\title{
Derepression of Cancer/Testis Antigens in cancer is associated with distinct patterns of DNA Hypomethylation
}

\author{
Robert Kim ${ }^{1,3}$, Prakash Kulkarni ${ }^{* *}$ and Sridhar Hannenhalli $i^{*}$
}

\begin{abstract}
Background: The Cancer/Testis Antigens (CTAs) are a heterogeneous group of proteins whose expression is typically restricted to the testis. However, they are aberrantly expressed in most cancers that have been examined to date. Broadly speaking, the CTAs can be divided into two groups: the CTX antigens that are encoded by the $X$-linked genes and the non-X CT antigens that are encoded by the autosomes. Unlike the non-X CTAs, the CTX antigens form clusters of closely related gene families and their expression is frequently associated with advanced disease with poorer prognosis. Regardless however, the mechanism(s) underlying their selective derepression and stage-specific expression in cancer remain poorly understood, although promoter DNA demethylation is believed to be the major driver.
\end{abstract}

Methods: Here, we report a systematic analysis of DNA methylation profiling data from various tissue types to elucidate the mechanism underlying the derepression of the CTAs in cancer. We analyzed the methylation profiles of 501 samples including sperm, several cancer types, and their corresponding normal somatic tissue types.

Results: We found strong evidence for specific DNA hypomethylation of CTA promoters in the testis and cancer cells but not in their normal somatic counterparts. We also found that hypomethylation was clustered on the genome into domains that coincided with nuclear lamina-associated domains (LADs) and that these regions appeared to be insulated by CTCF sites. Interestingly, we did not observe any significant differences in the hypomethylation pattern between the CTAs without CpG islands and the CTAs with CpG islands in the proximal promoter.

Conclusion: Our results corroborate that widespread DNA hypomethylation appears to be the driver in the derepression of CTA expression in cancer and furthermore, demonstrate that these hypomethylated domains are associated with the nuclear lamina-associated domains (LADS). Taken together, our results suggest that wide-spread methylation changes in cancer are linked to derepression of germ-line-specific genes that is orchestrated by the three dimensional organization of the cancer genome.

Keywords: DNA hypomethylation, Cancer/Testis antigens, Lamina attachment domains, Insulator regions

\footnotetext{
* Correspondence: pkulkar4@jhmi.edu; sridhar@umiacs.umd.edu 1James Buchanan Brady Urological Institute, The Johns Hopkins University School of Medicine, 600 North Wolfe Street, Baltimore, MD 21287, USA ${ }^{2}$ Department of Cell Biology \& Molecular Genetics, and Center for Bioinformatics \& Computational Biology, University of Maryland, 3104G Biomolecular Sciences Building (\#296), College Park, MD, USA

Full list of author information is available at the end of the article
} 


\section{Background}

The Cancer/Testis Antigens (CTAs) are a group of tumorassociated proteins that are typically expressed in normal male germ cells but are silent in normal somatic cells. However, they are aberrantly expressed in several types of cancers [1,2]. Because of this unique expression pattern, the CTAs are considered attractive targets for cancer biomarkers and immunotherapy [3].

Broadly speaking, the CTAs can be divided into two groups: the CTX antigens that are encoded by the $\mathrm{X}$ chromosome and the non-X CT antigens that are encoded by the autosomes. To date, 228 CTAs have been identified of which 120 CTAs (52\%) map to the X chromosome (the CTX antigens) while the remaining (non-X CT antigens), are distributed on the 22 autosomes and the Y chromosome [4]. Interestingly, while some autosomes that are gene-poor such as chromosome 21 (only 425 genes), are enriched for CTA genes (1.6 CTAs/100 genes), others, that are generich, such as chromosome 1 (3380 genes) and 7 (1764 genes), are very CTA-poor with only 0.3 CTAs and 0.06 CTAs/100 genes, respectively. However, among the sex chromosomes, while only 1 CTA is present on the Y chromosome, there are $7.5 \mathrm{CTAs} / 100$ genes on the $\mathrm{X}$ chromosome - a 125-fold increase over chromosome 7 [4].

Furthermore, the CTX antigens are comprised of large gene families of closely related members and are frequently associated with advanced disease with poorer prognosis [5-10]. It is remarkable that as much as $10 \%$ of the genes on the $\mathrm{X}$ chromosome are estimated to belong to CT-X families [11]. Although the role of many of these tumor-associated antigens in the disease process remains unclear, emerging evidence indicates that they appear to function in several important cellular processes such as transcriptional regulation, signal transduction, and cell growth [3]. Some also appear to function as putative proto-oncogenes $[12,13]$ and are associated with maintaining the undifferentiated state of stem cells [14-17].

More recently, a majority of the CTAs, especially the CTX antigens, were predicted to be intrinsically disordered proteins or IDPs [4]. IDPs are proteins that lack a rigid structure at least in vitro. Despite the lack of structure, most IDPs can transition from disorder to order upon binding to biological targets and often promote highly promiscuous interactions. Thus, IDPs play important roles in transcriptional regulation and signaling via regulatory protein networks and are frequently over-expressed in pathological conditions such as cancer $[18,19]$. Consistent with these observations, several CTAs are predicted to bind to DNA and their forced expression appears to increase cell growth implying a potential dosage-sensitive function [4]. Taken together, these observations provide a novel perspective on the CTAs implicating them in processing and transducing information in altered physiological states in a dosage-sensitive manner. Thus, understanding how the CTAs are selectively derepressed in cancer is an important question in cancer biology.

Although the mechanism promoting their derepression is not entirely clear, it is widely held that DNA methylation is one of the central mechanisms responsible for gene silencing [20-22]. For example, De Smet et al. have observed selective and genome-wide hypomethylation of MAGE-A1, one of the most studied CTAs in cancer cells, coincided with its activation [23-25]. Several other studies have also reported a similar trend in other CTA genes [26-30]. Roman-Gomez et al. discovered direct correlation between the methylation levels of the HAGE gene and its expression in myeloid leukemia [29]. Similarly, Cho et al. observed expression of the CAGE gene and its promoter hypomethylation in gastric cancer [27]. Yegnasubramanian et al. found that although the CT-X antigens undergo DNA hypomethylation and overexpression in primary prostate cancers, these changes were more pronounced in metastatic disease when many CT-X antigens were highly upregulated coincident with poorer prognosis [30]. Consistent with this hypothesis, other studies have shown that inhibiting DNA methyltransferase (DNMT) activity with 5 aza-deoxycytidine (5 AZA) results in robust somatic expression of a set of CTAs both in vitro and in vivo [31]. However, only a few studies have experimentally confirmed promoter demethylation following DNMT inhibition by 5AZA or silencing by siRNA [13,32] and in many cases CTA genes that lack CpG dinucleotides respond to DNMT inhibition while in other cases, despite the presence of $\mathrm{CpG}$ dinucleotides, the CTA genes are not derepressed. For instance, the SPANX genes, which lack a CpG island in the promoter region [33], respond robustly to 5 AZA treatments [34] implicating an indirect mechanisms underlying the response, although the presence of such sites at distal regions or within introns cannot be ruled out. It is therefore unclear to what extent these effects are mediated directly by promoter demethylation of the target genes as opposed to being indirectly driven by demethylation in conjunction with transcription factors that activate CTA expression.

Thus, it is obvious that our understanding of CTA gene regulation and mechanisms underlying their abrupt derepression in cancer has been not subjected to a genomewide analysis to assess their generality. Such a genome-wide analysis has recently become possible due to availability of genome-scale methylation arrays and other related technologies. Here, using genome-scale methylation profiles of promoter CpG methylation in 501 samples that included 305 normal sperm and somatic cells, and 196 cancers, we employed a new metric to identify gene promoters that follow an expected pattern of CTA promoter methylation, i.e., promoters that are unmethylated in sperm, methylated in somatic tissues, but unmethylated in cancer tissues. The higher the metric value for a gene 
promoter, the more closely it follows the prototypical methylation pattern (PMP). Our analysis confirmed that CTA gene promoters broadly follow a PMP. At the genome level, we observed that PMP promoters tend to cluster together on the genome and the CTA genes appeared to strongly associate with such clusters. Furthermore, we discovered that the binding sites for CTCF, the generic 'insulator protein', demarcate the regions of PMP. Genomic regions with PMPs have been observed to be enriched for genes involved in defense response, immune response, and cytokine-cytokine receptor interaction [35,36]. We also found that a large fraction of CTAs genes, especially the ones associated with clusters of PMPs coincided with the nuclear lamina-associated domains (LADs). However, we did not observe any significant differences in the above hypomethylation patterns between the promoters with CpG islands (CGI) and promoters without CpG islands (non-CGI). Taken together, our results indicate that PMP is a broad phenomenon covering CTAs and that their derepression is significantly explained by previously observed broad domains of hypomethylation in cancer that are associated with LADs.

\section{Methods}

\section{Methylation data}

DNA methylation profiling data were obtained from the Gene Expression Omnibus (GEO) database [37]. To be consistent, methylation profile datasets from only one platform, Illumina HumanMethylation27 BeadChip (GPL8490) containing 27578 genome-wide promoter CG dinucleotides, was used in this study. Our methylation dataset contained 501 samples from profiling studies for five different tissues and conditions: breast cancer tissues (GSE26990), colorectal cancer tissues (GSE25062, GSE17648), normal sperm (GSE26974), and prostate cancer tissues (GSE26126). The processed and normalized data was used as provided. The CG loci were partitioned into two groups based on whether it belonged to a $\mathrm{CpG}$ islands (CGIs; 16561 loci) or not (non-CGIs; 11017 loci). Human CGIs were obtained from the UCSC Genome Browser (genome.ucsc.edu; Build 36, hg18).

\section{Estimating delta-PMP for a CTCF site}

For each CTCF location, three nearest genes (by their transcription start sites) 5 ' of the CTCF site and three nearest genes 3' of the CTCF site were determined using ENSEMBL gene annotation (www.ensembl.org). A CTCF site was included for the analysis, if at least one of the three promoters to the left of the CTCF site and at least one of the three promoters to the right of the site had a CG locus represented in the methylation dataset. We computed the average PMP-sim for the promoters to the left and average PMP-sim for the promoters to the right. Then, we computed the absolute difference of those two averages as delta-PMP. The above procedure was performed separately for CGI loci and non-CGI loci.

\section{Results}

\section{Method overview}

Table 1 provides a summary of the Gene Expression Omnibus (GEO) datasets used in this study. Overall, the datasets included 501 distinct samples across $27578 \mathrm{CpG}$ loci in the genome based on the HumanMethylation27 BeadChip data (Illumina, CA). Out of 501 samples, 289 (58\%) of them were from tumors, while 212 (42\%) were normal. Thus, the data is summarized as a matrix with 27578 rows and 501 columns with methylation intensity. As shown in Figure 1, we defined a prototypical methylation pattern (PMP) vector with 501 entries, one per sample, as follows. For each sample $i$, (1) if the sample was obtained from either normal sperm cells or a cancer tissue, then the minimum methylation value of all the genes for that sample was assigned to the $i^{\text {th }}$ entry in PMP vector, and (2) if the sample was obtained from a normal somatic tissue, then the maximum methylation value of all the genes for that sample was assigned to the $i^{t h}$ entry in PMP vector. The PMP vector values are reported in Additional file 1: Table S1.

To quantify how well a particular CpG location $j$ conforms to the PMP, we computed the Pearson correlation between the $j^{t h}$ row and the PMP-vector; we refer to this as PMP-sim or $S_{j}$. A high value of $S_{j}$, indicates that the CpG location is methylated in normal somatic cells and unmethylated in sperm and cancer cells.

\section{CTA and CTX gene promoters have prototypical methylation patterns}

We computed the PMP-sim for all 27578 loci of which 92 correspond to non-X CTA genes (hereon referred to as CTA) and 47 correspond to CTX genes. Overall, the average PMP-sim values was $-0.014 \pm 0.17$, while for the CTA and the CTX genes the average PMP-sim was $0.18 \pm 0.17$ and $0.27 \pm 0.087$ respectively (Figure 2 ). The difference between CTA and all genes was highly significant with Mann-Whitney $U$ test $p$-value $=1.47 \mathrm{E}-21$, and likewise for CTX versus all genes with $p$-value $=1.16 \mathrm{E}-23$. Furthermore, the difference between CTX and CTA was also significant ( $p$-value $=0.002)$. We have provided a heatmap (Additional file 2: Figure S1) which clearly shows the prototypical methylation patterns of CTA and CTX genes across 501 samples. Furthermore, we have also demonstrated that several well-known CTAs (MAGE, XAGE, PAGE, and GAGE families) follow the prototypical pattern closely (Additional file 3: Figure S2). All four families had significantly high PMP-sim values: MAGE family $(n=19$; PMP-sim $=0.27 \pm$ 0.092), XAGE family $(n=2$; PMP-sim $=0.22 \pm 0.045)$, PAGE family $(n=4$; PMP-sim $=0.22 \pm 0.052)$, and GAGE family $(n=2$; PMP-sim $=0.30 \pm 0.038)$. Our conclusion does not change when we constructed the PMP vector by assigning 
Table 1 GEO methylation studies included for analysis

\begin{tabular}{ccccc}
\hline GEO accession & Tissue type & Total samples & Normal samples & Tumor samples \\
\hline GSE26990 & Breast** & 89 & 42 & 47 \\
GSE26126 & Prostate & 193 & 98 & 95 \\
GSE17648 & Colorectal & 44 & 22 & 22 \\
GSE25062 & Colorectal & 154 & 29 & 125 \\
GSE26974 & Sperm & 21 & 21 & 0 \\
\hline
\end{tabular}

All methylation profiling was done based on Illumina HumanMethylation27 BeadChip (GEO platform GPL8490).

** Also included nine normal samples from other tissues (blood, spleen, colon, fetal brain, liver, testis, embryo, and adult brain).

the value of 0 to sperm and cancer cells and the value of 1 to normal somatic cells.

In genome-wide profiling of gene expression and other studies such as DNA methylation, laboratory-specific biases are a significant concern. A visual inspection of the methylation profiles organized by GEO series indicated such a bias. To ensure that our conclusion regarding a greater PMP-sim value for CTA and CTX is not simply because of this bias, we performed the following control. For each CG locus, within each GEO series (Table 1), we randomly permuted the samples. This has the effect of randomizing the normal/tumor identity of the sample while preserving the lab-specific (series-specific) biases. If our observed results above are primarily due to laboratory-specific biases then we would expect the PMP-sim values to be largely preserved. We found this not to be the case. While overall, the PMP-sim did not change significantly (going from $0.014 \pm$ 0.17 for original data to $0.01 \pm 0.10$ for the permuted data), the PMP-sim for CTA loci was significantly reduced from $0.18 \pm 0.17$ to $0.03 \pm 0.11$ and that for the CTX genes significantly reduced from $0.27 \pm 0.09$ to $0.06 \pm 0.10$. Thus, we conclude that our observed elevated PMP-sim for CTA/ CTX loci is not simply due to laboratory-specific biases.

Next, to assess the robustness of our findings above, of the 501 total samples, we randomly sampled 70 samples: 10 normal breast samples (GSE26990), 10 breast cancer samples (GSE26990), 10 normal prostate samples (GSE26126), 10 prostate cancer samples (GSE26126), 10

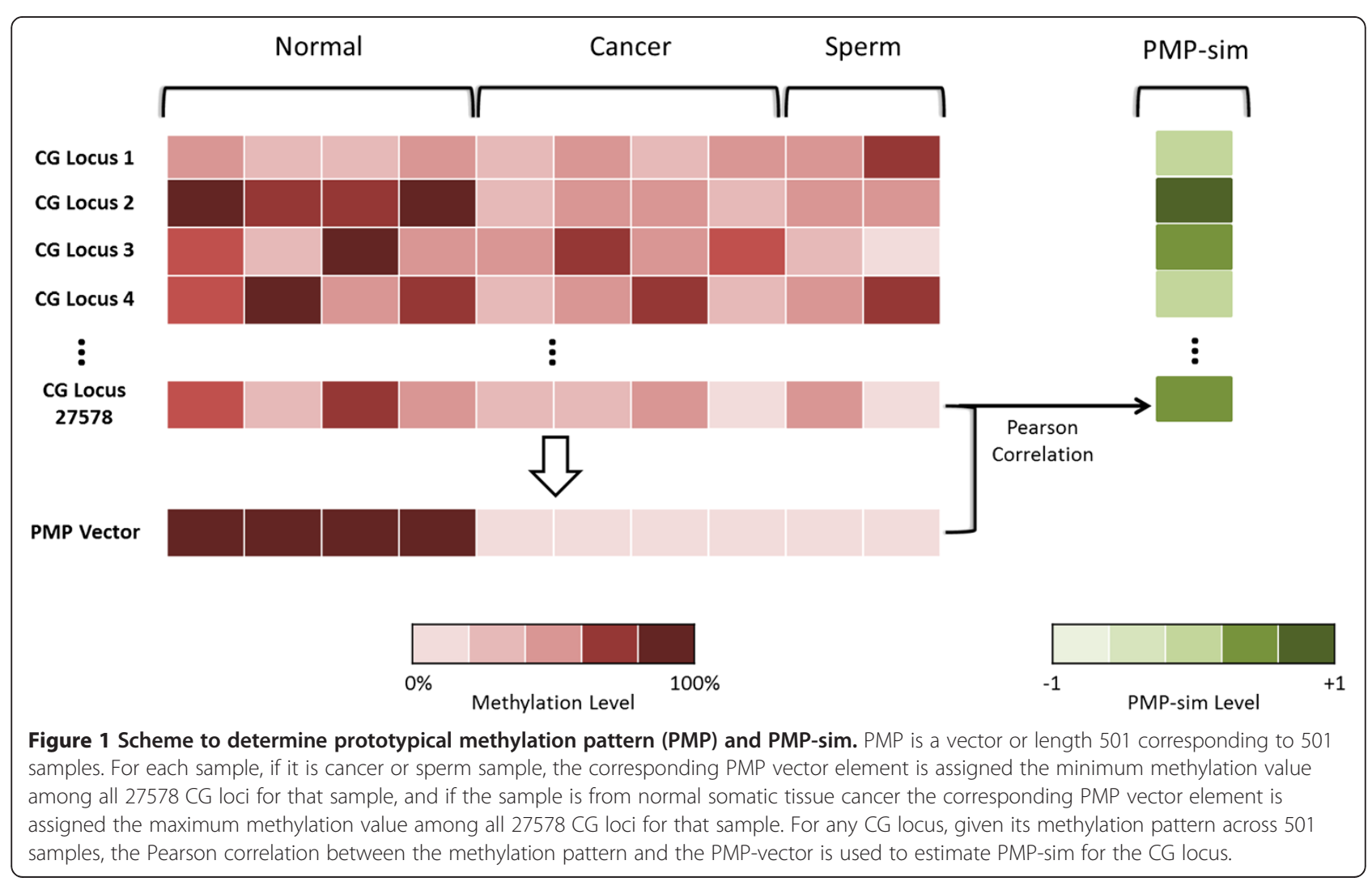



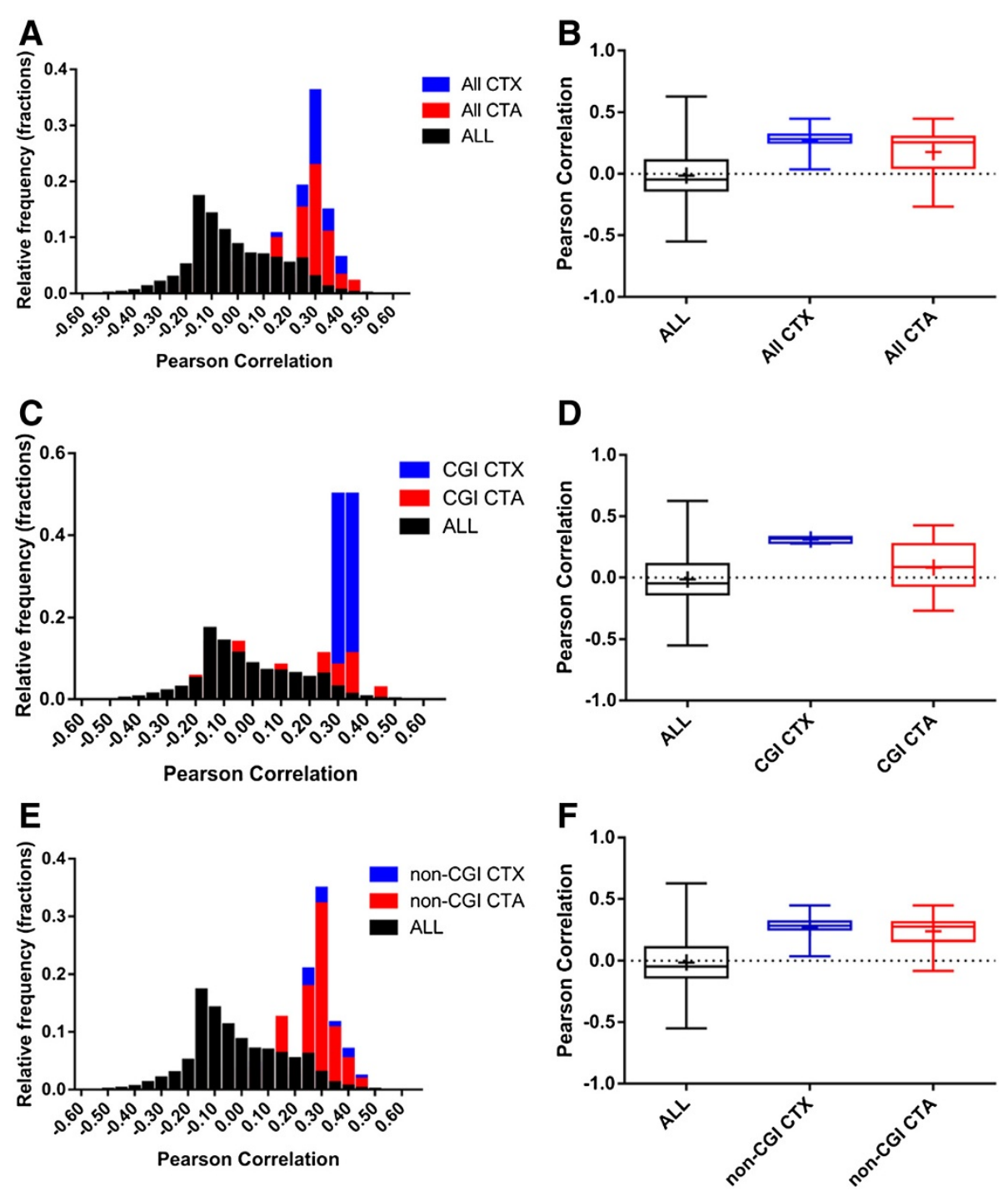

Figure 2 Pearson correlation values for the CTX and CTA genes are significantly high. (A-B) Pearson correlation distribution in three groups: all the loci $(n=27578)$, CTX loci $(n=47)$, and CTA loci $(n=92)$. A box and whisker plot comparing the correlation values among the three loci groups is shown in (B). The plot shows the median, the mean (crosses inside the boxes), $25^{\text {th }}$ percentile (bottom line of the box), $75^{\text {th }}$ percentile (top line of the box), and minimum and maximum values as whiskers. (C-D) Pearson correlation distribution in three groups: all the loci ( $n=27578)$, CTX loci in CGI regions $(n=4)$, and CTA loci in CGI regions $(n=36)$. (E-F) Pearson correlation distribution in three groups: all the loci $(n=27578)$, CTX loci in non-CGl regions $(n=43)$, and CTA loci in non-CGl regions $(n=56)$.

normal sperm samples (GSE26974), 10 normal colorectal samples (5 from GSE17648 and 5 from GSE25062), and 10 colorectal cancer samples (5 from GSE17648 and 5 from GSE25062). Using these randomly chosen 70 samples instead of 501 samples as above, we computed the PMP-sim for all 27578 loci again and observed high PMPsim values for the CTA and CTX genes. The results were highly consistent with those obtained when using all 501 samples. The average PMP-sim value across all the loci decreased slightly (going from $0.014 \pm 0.17$ for the original data to $-0.003 \pm 0.21$ for the re-sampled data). The average PMP-sim values for the CTA and CTX genes were $0.18 \pm 0.18(0.18 \pm 0.17$ for the original data $)$ and $0.25 \pm 0.09$
$(0.27 \pm 0.09$ for the original data), respectively. Thus, the results support the robustness of the greater PMP-sim observed for the CTA/CTX loci.

We then partitioned the 27578 promoter CG dinucleotides into 16561 that resided within a CpG island (CGI) and the rest 11017 that did not (non-CGI). Corresponding CGI and non-CGI, counts for CTA genes were 36 and 56 and those for CTX were 4, and 43, respectively. We repeated the above analyses separately for CGI and non-CGI promoters and the results were similarly significant (Figure 2): all genes vs. CGI CTA (Mann-Whitney $U$ test $p$-value $=0.0048)$, all genes vs. CGI CTX $(p$-value $=0.0011)$, all genes vs. non-CGI CTA ( $p$-value $=2.00 \mathrm{E}-23)$, and all 
genes vs. non-CGI CTX ( $p$-value $=2.34 \mathrm{E}-21)$. Thus, our results suggest that CTA promoters largely follow a PMP across various tissues in both CGI and non-CGI promoters. However, the number of CGI CTX loci was very small, even though the tests showed statistical significance.

\section{Promoters that follow prototypical methylation pattern are clustered on the genome}

To further understand the mechanism underlying the PMP, we next tested whether the CG dinucleotides that follow PMP, i.e. have high PMP-sim, are clustered on the genome. We identified CG dinucleotides with PMP-sim in top $20^{\text {th }}$ percentile; we refer to this set as high-PMP. We constructed a binary vector of length 27578 corresponding to all CG dinucleotides sorted by their genomic locations. We assigned a ' 1 ' at locations corresponding to high-PMP CGs and ' 0 ' to the rest. In this binary vector, a run was defined as consecutive ' 1 's and the length of a run as the number of consecutive ones in the vector. We identified the runs and their lengths separately for CGIs and nonCGIs promoters. Long runs are suggestive of genomic clustering of PMP promoters. As a control we randomly permuted the binary vector. As shown in Figure 3, we found that for both CGIs and non-CGI promoters the run lengths were significantly higher than that for the corresponding controls (Mann-Whitney $U$ test $p$-value $=$
0.0001 for CGIs and 1.47E-12 for non-CGIs). The average run length for CGIs was $2.348 \pm 0.722$ (range $1-9$ ), and $1.241 \pm 0.533$ (range $1-5$ ) in permuted control. The average run length for non-CGIs was slightly higher at $2.669 \pm$ 1.358 (range 1 - 15) and the corresponding control had run lengths $1.254 \pm 0.556$ (range $1-4$ ). CGIs had 633 runs (26\%) with length of two or greater out of 2460 runs. NonCGIs had 429 runs (28\%) with length of two or greater out of 1488 runs. These results, summarized in Figure 3, suggest that promoters with PMP are clustered on the genome for both CGI and non-CGI promoters.

\section{CTA and CTX gene promoters reside largely within PMP runs}

Next, we assessed the extent to which CTA and CTX genes reside within runs of promoters with PMP. As shown in Table 2 for both CGIs and non-CGIs, the fractions of CTA and CTX genes residing within runs were significantly higher than their control groups (binary vectors from runof-ones analysis permuted 100 times). All comparisons using $\mathrm{z}$-score statistics yielded $p$-value $<4.72 \mathrm{E}-12$. It is also interesting to note that the fractions of CTA and CTX loci that are part of a run (referred to as CTA-F and CTX-F in Table 2) from non-CGIs were far greater than those from CGIs. In other words, more CTA and CTX genes from non-CGIs comprised the runs of length two or greater.
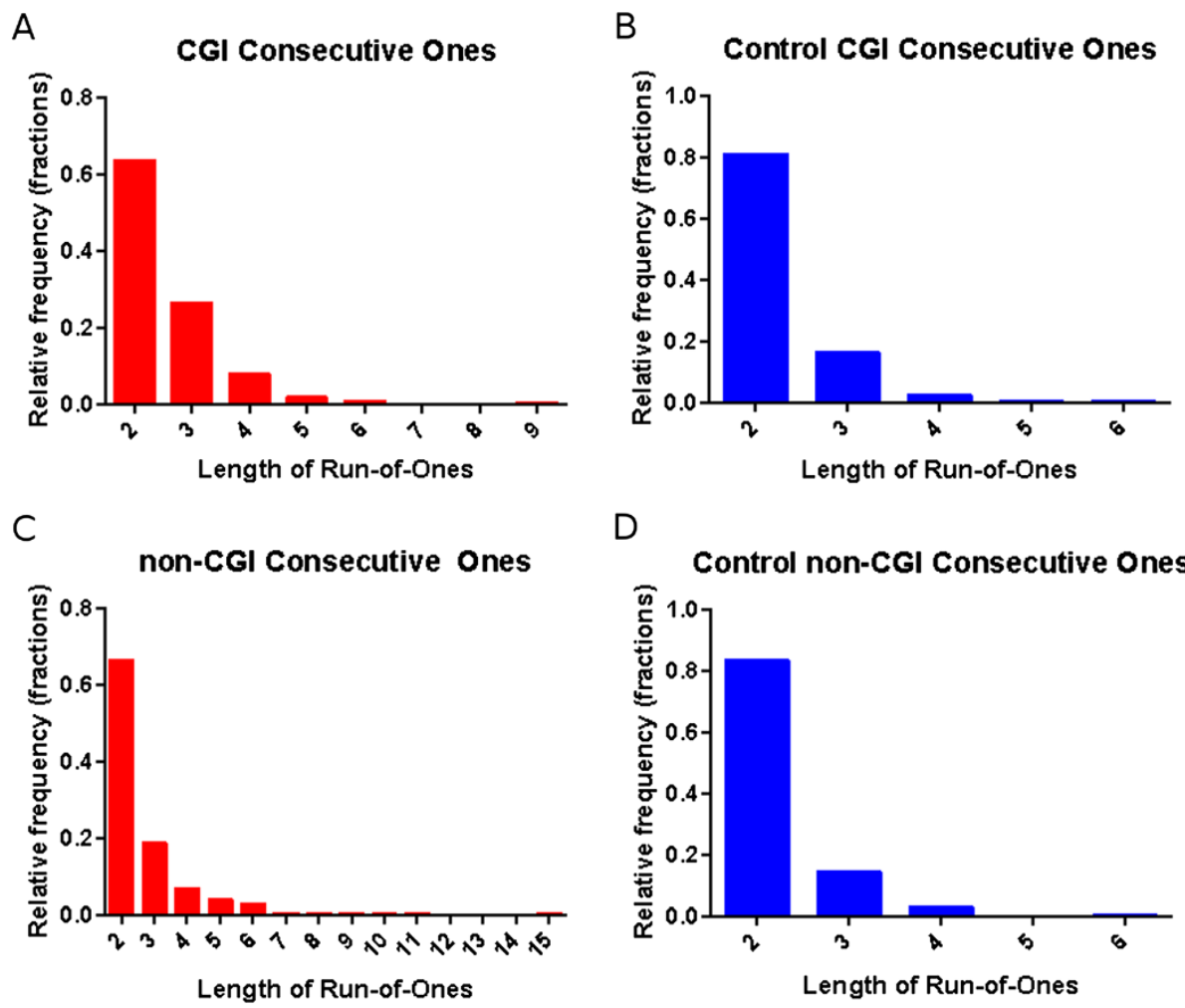

Figure 3 PMP run lengths for CGls and non-CGls were higher than the random control. Distribution of runs with length of two or greater are shown for (A) CGls, (B) randomized CGls as a control group for (A), (C) non-CGls, and (D) randomized non-CGls as a control group for (C). 
CTCF binding sites demarcate PMP from non-PMP regions CCCTC-binding factor (CTCF) is a multifunctional protein best known for its role as an insulator of epigenomic marks $[38,39]$. Thus, we asked whether the presence of CTCF binding sites has any bearing on PMP at consecutive CG loci intervened by CTCF binding. A previous study comparing CTCF binding in multiple cell types had shown that a large fraction of CTCF binding events are conserved across cell types [40]. Thus, in our analysis we only included 7428 CTCF sites that were common to 4 cell types, namely CD4+ T cell, IMR90, Hela, and Jurkat. Details of individual datasets and extraction method are provided in [40]. For each CTCF binding site we assessed its insulator tendency as the absolute difference in average PMP-sim between 3 CG loci to the 5' of the CTCF site and 3 CG loci to the 3' of the CTCF site (see Methods); we refer to this value as delta-PMP. As a control for CTCF sites, we chose random loci in the genome.

Interestingly, we found that for both CGIs and nonCGIs promoters, the delta-PMP for CTCF sites were significantly higher than the random control (Figure 4). The average delta-PMP for CGIs was $0.12 \pm 0.09$, while the random delta-PMP was $0.062 \pm 0.05$ (Mann-Whitney $U$ test $p$-value $=1.38 \mathrm{E}-217)$. A similar trend was observed in non-CGIs; the mean CTCF delta was $0.12 \pm 0.09$, while the mean random delta was $0.07 \pm 0.05$ (Mann-Whitney $U$ test $p$-value $=7.59 \mathrm{E}-52$ ). Moreover, the delta-PMP distribution of CGIs and non-CGIs was statistically indistinguishable. Further, when we tested whether the CTAs or runs of CTAs reside near a CTCF site relative to random expectation, we found this not to be the case, suggesting that potential involvement of CTCF sites in demarcating PMP regions is not relevant to CTAs and is instead a general phenomenon.

\section{Promoters with PMP intersect with Lamin Attachment Domains (LADs)}

Regions of hypomethylation in cancer have previously been shown to significantly intersect with LADs and are thus

Table 2 Fractions of CTA and CTX genes in CGIs and non-CGIs that are in runs with length of two or greater

\begin{tabular}{|c|c|c|c|}
\hline \multicolumn{4}{|c|}{ CGls } \\
\hline \multicolumn{2}{|c|}{ Original Binary Vector } & \multicolumn{2}{|c|}{$\begin{array}{c}\text { Permuted Binary Vector } \\
\text { (avg. } \pm \text { sd.) }\end{array}$} \\
\hline CTA-F & CTX-F & CTA-F & CTX-F \\
\hline 0.250 (9 out of 36 ) & 1 (4 out of 4$)$ & $0.013 \pm 0.0248$ & $0.0175 \pm 0.0815$ \\
\hline \multicolumn{4}{|c|}{ Non-CGls } \\
\hline \multicolumn{2}{|c|}{ Original Binary Vector } & \multicolumn{2}{|c|}{ Permuted Binary Vector } \\
\hline CTA-F & CTX-F & CTA-F & CTX-F \\
\hline 0.786 (44 out of 56 ) & 0.884 (38 out of 43$)$ & $0.226 \pm 0.081$ & $0.223 \pm 0.077$ \\
\hline
\end{tabular}

thought to be critical in organizing the interphase chromosomes [41]. We extracted 1344 LAD loci from [42]. Out of 5397 high-PMP (CGI and non-CGI loci combined) 1389 (25.74\%) resided within a LAD, and of the 21558 other CG loci only 3229 (14.98\%) resided within a LAD. This difference was statistically highly significant (Fisher's exact test $p$-value $=8.17 \mathrm{E}-50$ ). The difference is similarly significant when CGI and non-CGI loci were analyzed separately. This result along with our finding that CTA (and CTX) CG loci have high PMP-sim would suggest a high correlation between CTAs and LADs. This is indeed the case and as shown in Figure 5, the fraction of CTA and CTX loci, both overall, as well as the ones that intersect with high-PMP runs, intersect with LAD regions five- to six-fold more frequently than random expectations: all comparisons using z-score statistics yielded an extremely small $p$-value (almost 0 ). In addition, we found that the CG loci within LADs had significantly greater PMP-sim values than the CG loci outside LADs (Mann-Whitney $U$ test $p$-value $=$ 8.20E-31). Thus, our results suggest that high-PMP runs, and consequently CTA and CTX loci with PMPs largely intersect with LAD regions.

Given that CTCF sites demarcate PMP from non-PMP regions, that PMP regions overlap with $\mathrm{LAD}$, and that $\mathrm{LAD}$ were previously shown to be demarcated by CTCF sites [42], we expect that LAD boundaries themselves demarcate PMP from non-PMP regions. Similar to our previous analysis performed on the CTCF binding sites, we assessed the tendency of LAD boundaries to exhibit high difference in PMP-sim for the three CG loci to the 5' and the three CG loci to the 3'. As expected we observed a trend similar to that observed for CTCF sites. The delta-PMP values for LAD boundary loci were significantly higher than the random control for both CGIs and non-CGIs promoters. The average delta-PMP for LAD loci in CGIs was $0.11 \pm 0.089$, while the control delta-PMP was $0.063 \pm 0.052$ (MannWhitney $U$ test $p$-value $=8.27 \mathrm{E}-30$ ). The average deltaPMP for LAD loci in non-CGIs was $0.11 \pm 0.094$, while its control delta-PMP was $0.071 \pm 0.055$ ( $p$-value $=2.43 \mathrm{E}-7$ ).

\section{Discussion}

Even amongst the so-called tissue-specific genes, the CTAs exhibit a remarkable expression pattern. While typically expressed only in the sperm and repressed in normal somatic tissues, they are aberrantly derepressed in most cancers [1]. However, neither the mechanism nor the functional consequence of this atypical expression pattern is entirely clear for most, if not all, CTAs. While there is evidence to suggest that promoter demethylation might be a major driver of derepression of CTA expression in cancer [3], this mechanism does not appear to be universally applicable to all CTAs [34]. Independent of CTA-related investigations, it has been shown that large genomic regions are hypomethylated in some cancers [41,43]. It is therefore 

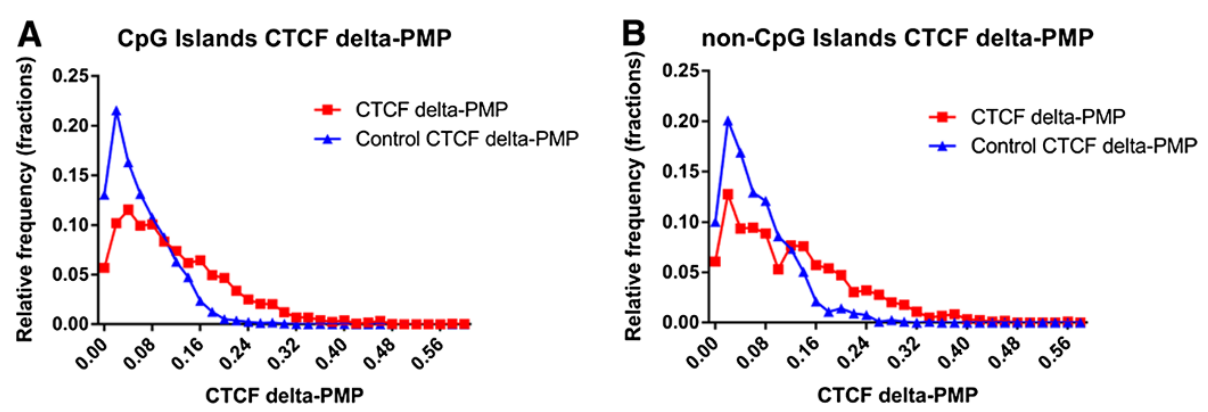

Figure 4 Delta-PMP values for the CTCF sites in CGIs and non-CGls were higher than the random control. (A) delta-PMP distribution for the CTCF sites in CGIs (red) and its control (blue). (B) delta-PMP distribution for the CTCF sites in non-CGls (red) and its control (blue).

tempting to speculate that CTAs may be swept by the global hypomethylation as bystanders leading to their derepression. Based on a systematic analysis of DNA methylation profiling data from various tissues, our results support this thesis.

We found specific hypomethylation of the CTA and CTX promoters in the testis and cancer cells. More specifically, we observed hypomethylation of MAGE, XAGE, PAGE, and GAGE promoters in cancer samples (Additional file 3: Figure S2) confirming several studies that have reported that the activation of these genes in cancer is strongly correlated with promoter demethylation $[23,44,45]$. This result, combined with well-established association between DNA methylation and gene silencing suggests methylation as the predominant mechanism for CTA derepression in cancers. Moreover, the loci with PMP including the ones in CTA and CTX promoters,

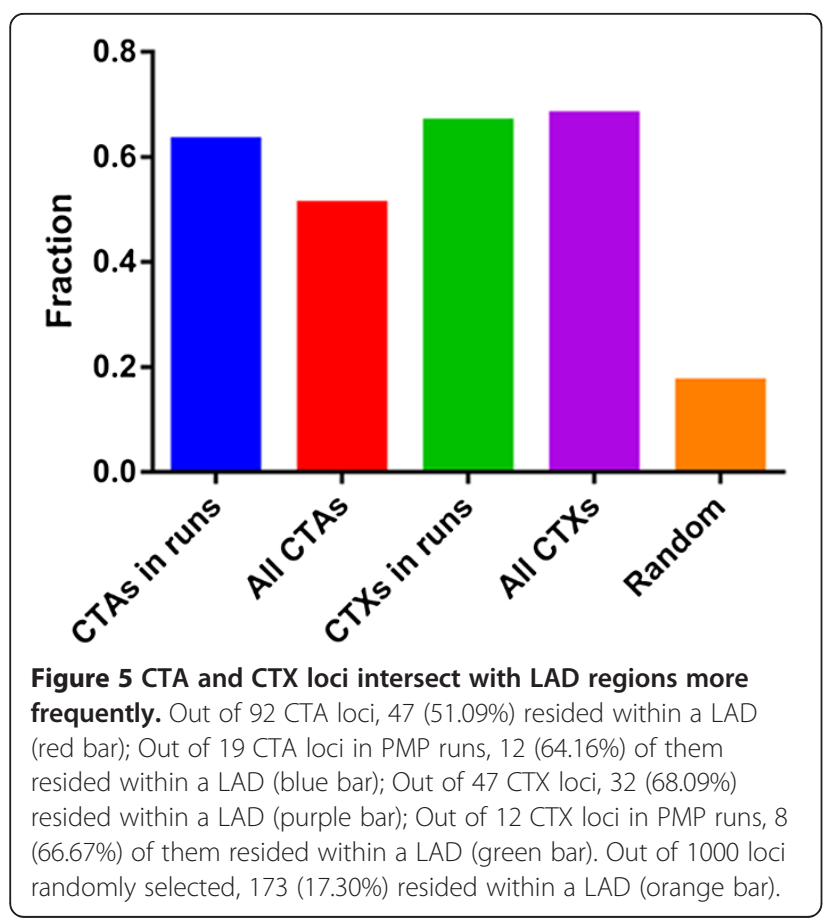

cluster on the genome and are associated with LAD regions. This is consistent with broad regions of hypomethylation in cancers that are also associated with LAD regions [41]. Taken together, these findings suggest that hypomethylation and derepression of CTA and CTX genes in cancers are part of a broader phenomenon and may not depend on a specific mechanism. We also found that the broad tendencies revealed by our analyses are independent of $\mathrm{CpG}$ islands. This may suggest a nonspecific mechanism underlying methylation-mediated derepression of CTA genes. Furthermore, we also found that CTCF sites are linked to a sudden change in methylation patterns for both CGIs and non-CGI loci. This is consistent with a previous study that found epigenetic silencing of tumor suppressor genes in the absence of CTCF binding [46].

We note that the methylation profiling platform (Illumina HumanMethylation27 BeadChip) used in this study includes only $1 \mathrm{CpG}$ locus per gene promoter, resulting in a small number loci corresponding to CTA and CTX genes. Furthermore, a single CpG site may not be representative of an entire promoter in all cases. Although Illumina has a newer and denser methylation chip (Illumina Human Methylation450 BeadChip) which contains more than 450,000 methylation sites, the number of relevant tissues for which such data exists is currently insufficient. In addition, although previous works have illustrated an inverse correlation between promoter methylation and gene expression level, we could not ascertain this for our data because none of the samples included in the study had a corresponding expression data available.

\section{Conclusions}

In conclusion, here we present a systematic analysis based on large number of genome-wide methylation profiles across multiple normal and tumor cells demonstrating that in general, derepression of CTA and CTX genes in cancer may be largely explained by global hypomethylation mediated by disruption of laminar attachment regions. However, it is important to note that, DNA hypomethylation may not 
be the only mechanism since several CTAs lacking CpG islands are also upregulated in response to DNA hypomethylation [47]. Thus, it is possible that at least for some CTA genes, additional mechanisms for repression and alternative mechanisms for derepression in cancer exist which may involve specific repressive or activating transcription factors. Additional biochemical studies elucidating global methylation changes should yield new insights on regulation of CTA expression paving the way to the development of novel therapeutic modalities for cancer. This is particularly important since epigenetic modulation of CTA expression is emerging as a novel medical modality for cancer immunotherapy $[32,48,49]$.

\section{Additional files}

Additional file 1: Table S1. The PMP vector for 501 samples is shown in the file.

Additional file 2: Figure S1. Heatmap of the methylation levels of CTA CTX, and non-CTA loci across 501 samples. Heatmap of methylation data shows the prototypical methylation patterns of CTA and CTX loci: high methylation levels (yellow in the heatmap) in normal samples and low methylation levels (green in the heatmap) in cancer and sperm cells. On the other hand, the methylation levels of 150 randomly selected nonCTA loci did not follow the prototypical methylation patterns.

Additional file 3: Figure S2. Heatmap of the methylation levels of MAGE, XAGE, PAGE, and GAGE promoter loci across 501 samples. Heatmap of methylation data shows that these four CTX families follow the prototypical methylation patterns: high methylation levels (yellow in the heatmap) in normal samples and low methylation levels (green in the heatmap) in cancer and sperm cells. The average PMP-sim values for the four families are: $0.27 \pm 0.092$ (MAGE family; $n=19$ ), $0.22 \pm 0.045$ (XAGE family; $n=2$ ), $0.22 \pm 0.052$ (PAGE family; $n=4$ ), and $0.30 \pm 0.038$ (GAGE family; $n=2)$. The PMP vector is shown in the bottom part of the figure (boxed), and the PMP-sim value for each gene is shown on the right vertical axis

\section{Abbreviations}

CTA: Cancer/Testis Antigen; CTX: Cancer/Testis X Antigen; CGI: CpG island; nCGI: non-CpG island; PMP: Prototypical methylation pattern; CTCF: CCCTCbinding factor; CTCFL: CTCF-like protein; LAD: Lamina associated domain.

\section{Competing interests}

The authors have no competing interests to declare.

\section{Authors' contribution}

The study was conceived by P.K. and S.H. All analysis was done by R.K. The manuscript was written jointly by all authors. All authors read and approved the final manuscript.

\section{Acknowledgement}

This work is supported by a grant from the David Koch Fund to PK and a $\mathrm{NIH}$ grant R01GM100335 to S.H. Authors thank Dr. Sebastien Vigneau and Dr. Steven Mooney for helpful comments on the manuscript. Publication of this article was funded in part by the Open Access Promotion Fund of the Johns Hopkins University Libraries.

\section{Author details}

'James Buchanan Brady Urological Institute, The Johns Hopkins University School of Medicine, 600 North Wolfe Street, Baltimore, MD 21287, USA. ${ }^{2}$ Department of Cell Biology \& Molecular Genetics, and Center for Bioinformatics \& Computational Biology, University of Maryland, 3104G Biomolecular Sciences Building (\#296), College Park, MD, USA. ${ }^{3}$ Present address: Department of Neurology, Yale University School of Medicine, New Haven, CT, USA.
Received: 4 January 2013 Accepted: 14 March 2013

Published: 22 March 2013

\section{References}

1. Scanlan MJ, Simpson AJ, Old LJ: The cancer/testis genes: review, standardization, and commentary. Cancer Immun 2004, 4:1.

2. Stevenson BJ, Iseli C, Panji S, Zahn-Zabal M, Hide W, Old LJ, Simpson AJ, Jongeneel CV: Rapid evolution of cancer/testis genes on the X chromosome. BMC Genomics 2007, 8:129.

3. Scanlan MJ, Gure AO, Jungbluth AA, Old LJ, Chen YT: Cancer/testis antigens: an expanding family of targets for cancer immunotherapy. Immunol Rev 2002, 188:22-32.

4. Rajagopalan K, Mooney SM, Parekh N, Getzenberg RH, Kulkarni P: A majority of the cancer/testis antigens are intrinsically disordered proteins. J Cell Biochem 2011, 112(11):3256-3267.

5. Andrade VC, Vettore AL, Felix RS, Almeida MS, Carvalho F, Oliveira JS, Chauffaille ML, Andriolo A, Caballero OL, Zago MA, et al: Prognostic impact of cancer/testis antigen expression in advanced stage multiple myeloma patients. Cancer Immun 2008, 8:2.

6. Grigoriadis A, Caballero OL, Hoek KS, da Silva L, Chen YT, Shin SJ, Jungbluth AA, Miller LD, Clouston D, Cebon J, et al: CT-X antigen expression in human breast cancer. Proc Natl Acad Sci USA 2009, 106(32):13493-13498.

7. Gure AO, Chua R, Williamson B, Gonen M, Ferrera CA, Gnjatic S, Ritter G, Simpson AJ, Chen YT, Old $L$, et al: Cancer-testis genes are coordinately expressed and are markers of poor outcome in non-small cell lung cancer. Clin Cancer Res 2005, 11(22):8055-8062.

8. Napoletano C, Bellati F, Tarquini E, Tomao F, Taurino F, Spagnoli G, Rughetti A, Muzii L, Nuti M, Benedetti Panici P: MAGE-A and NY-ESO-1 expression in cervical cancer: prognostic factors and effects of chemotherapy. Am J Obstet Gynecol 2008, 198(1):99. e91-97.

9. Suyama T, Shiraishi T, Zeng Y, Yu W, Parekh N, Vessella RL, Luo J, Getzenberg RH, Kulkarni P: Expression of cancer/testis antigens in prostate cancer is associated with disease progression. Prostate 2010, 70(16):1778-1787.

10. Velazquez EF, Jungbluth AA, Yancovitz M, Gnjatic S, Adams S, O'Neill D, Zavilevich K, Albukh T, Christos P, Mazumdar M, et al: Expression of the cancer/testis antigen NY-ESO-1 in primary and metastatic malignant melanoma (MM)-correlation with prognostic factors. Cancer Immun 2007, 7:11.

11. Ross MT, Grafham DV, Coffey AJ, Scherer S, McLay K, Muzny D, Platzer M, Howell GR, Burrows C, Bird CP, et al: The DNA sequence of the human X chromosome. Nature 2005, 434(7031):325-337.

12. Cheng YH, Wong EW, Cheng CY: Cancer/testis (CT) antigens, carcinogenesis and spermatogenesis. Spermatogenesis 2011, 1(3):209-220

13. Smith IM, Glazer CA, Mithani SK, Ochs MF, Sun W, Bhan S, Vostrov A, Abdullaev Z, Lobanenkov V, Gray A, et al: Coordinated activation of candidate proto-oncogenes and cancer testes antigens via promoter demethylation in head and neck cancer and lung cancer. PLoS One 2009, 4(3):e4961

14. Bera TK, Saint Fleur A, Ha D, Yamada M, Lee Y, Lee B, Hahn Y, Kaufman DS, Pera M, Pastan I: Selective POTE paralogs on chromosome 2 are expressed in human embryonic stem cells. Stem Cells Dev 2008, 17(2):325-332.

15. Cronwright G, Le Blanc K, Gotherstrom C, Darcy P, Ehnman M, Brodin B: Cancer/testis antigen expression in human mesenchymal stem cells: down-regulation of SSX impairs cell migration and matrix metalloproteinase 2 expression. Cancer Res 2005, 65(6):2207-2215.

16. Gjerstorff MF, Harkness L, Kassem M, Frandsen U, Nielsen O, Lutterodt M, Mollgard K, Ditzel HJ: Distinct GAGE and MAGE-A expression during early human development indicate specific roles in lineage differentiation. Hum Reprod 2008, 23(10):2194-2201.

17. Lifantseva N, Koltsova A, Krylova T, Yakovleva T, Poljanskaya G, Gordeeva O Expression patterns of cancer-testis antigens in human embryonic stem cells and their cell derivatives indicate lineage tracks. Stem Cells Int 2011, 2011:795239.

18. Uversky VN, Oldfield CJ, Dunker AK: Intrinsically disordered proteins in human diseases: introducing the D2 concept. Annu Rev Biophys 2008, 37:215-246.

19. Vavouri T, Semple JI, Garcia-Verdugo R, Lehner B: Intrinsic protein disorder and interaction promiscuity are widely associated with dosage sensitivity. Cell 2009, 138(1):198-208. 
20. Das PM, Singal R: DNA methylation and cancer. J Clin Oncol 2004, 22(22):4632-4642.

21. Ehrlich M: DNA methylation in cancer: too much, but also too little. Oncogene 2002, 21(35):5400-5413.

22. Ramchandani S, MacLeod AR, Pinard M, von Hofe E, Szyf M: Inhibition of tumorigenesis by a cytosine-DNA, methyltransferase, antisense oligodeoxynucleotide. Proc Natl Acad Sci USA 1997, 94(2):684-689.

23. De Smet C, De Backer O, Faraoni I, Lurquin C, Brasseur F, Boon T: The activation of human gene MAGE-1 in tumor cells is correlated with genome-wide demethylation. Proc Natl Acad Sci USA 1996 93(14):7149-7153.

24. De Smet C, Loriot A, Boon T: Promoter-dependent mechanism leading to selective hypomethylation within the $5^{\prime}$ region of gene MAGE-A1 in tumor cells. Mol Cell Biol 2004, 24(11):4781-4790.

25. Loriot A, De Plaen E, Boon T, De Smet C: Transient down-regulation of DNMT1 methyltransferase leads to activation and stable hypomethylation of MAGE-A1 in melanoma cells. J Biol Chem 2006, 281(15):10118-10126.

26. Ademuyiwa FO, Bshara W, Attwood K, Morrison C, Edge SB, Ambrosone CB, O'Connor TL, Levine EG, Miliotto A, Ritter E, et al: NY-ESO-1 cancer testis antigen demonstrates high immunogenicity in triple negative breast cancer. PLoS One 2012, 7(6):e38783.

27. Cho B, Lee $H$, Jeong S, Bang YJ, Lee HJ, Hwang KS, Kim HY, Lee YS, Kang $\mathrm{GH}$, Jeoung DI: Promoter hypomethylation of a novel cancer/testis antigen gene CAGE is correlated with its aberrant expression and is seen in premalignant stage of gastric carcinoma. Biochem Biophys Res Commun 2003, 307(1):52-63.

28. Glazer CA, Smith IM, Ochs MF, Begum S, Westra W, Chang SS, Sun W, Bhan S, Khan Z, Ahrendt S, et al: Integrative discovery of epigenetically derepressed cancer testis antigens in NSCLC. PLoS One 2009, 4(12):e8189.

29. Roman-Gomez J, Jimenez-Velasco A, Agirre X, Castillejo JA, Navarro G, San Jose-Eneriz E, Garate L, Cordeu L, Cervantes F, Prosper F, et al: Epigenetic regulation of human cancer/testis antigen gene, HAGE, in chronic myeloid leukemia. Haematologica 2007, 92(2):153-162.

30. Yegnasubramanian S, Haffner MC, Zhang Y, Gurel B, Cornish TC, Wu Z, Irizarry RA, Morgan J, Hicks J, DeWeese TL, et al: DNA hypomethylation arises later in prostate cancer progression than CpG island hypermethylation and contributes to metastatic tumor heterogeneity. Cancer Res 2008, 68(21):8954-8967.

31. De Smet $C$, Lurquin $C$, Lethe $B$, Martelange $V$, Boon T: DNA methylation is the primary silencing mechanism for a set of germ line- and tumorspecific genes with a CpG-rich promoter. Mol Cell Biol 1999, 19(11):7327-7335.

32. Karpf AR: A potential role for epigenetic modulatory drugs in the enhancement of cancer/germ-line antigen vaccine efficacy. Epigenetics 2006, 1(3):116-120

33. Zendman AJ, Zschocke J, van Kraats AA, de Wit NJ, Kurpisz M, Weidle UH, Ruiter DJ, Weiss EH, van Muijen GN: The human SPANX multigene family: genomic organization, alignment and expression in male germ cells and tumor cell lines. Gene 2003, 309(2):125-133.

34. Menendez L, Walker D, Matyunina LV, Dickerson EB, Bowen NJ, Polavarapu $\mathrm{N}$, Benigno BB, McDonald JF: Identification of candidate methylationresponsive genes in ovarian cancer. Mol Cancer 2007, 6:10.

35. da Huang W, Sherman BT, Lempicki RA: Systematic and integrative analysis of large gene lists using DAVID bioinformatics resources. Nat Protoc 2009, 4(1):44-57.

36. da Huang W, Sherman BT, Lempicki RA: Bioinformatics enrichment tools: paths toward the comprehensive functional analysis of large gene lists. Nucleic Acids Res 2009, 37(1):1-13

37. Barrett T, Troup DB, Wilhite SE, Ledoux P, Rudnev D, Evangelista C, Kim IF, Soboleva A, Tomashevsky M, Marshall KA, et al: NCBI GEO: archive for highthroughput functional genomic data. Nucleic Acids Res 2009, 37(Database issue):D885-D890.

38. Dunn $\mathrm{KL}$, Davie JR: The many roles of the transcriptional regulator CTCF. Biochem Cell Biol 2003, 81(3):161-167.

39. Ohlsson $R$, Renkawitz $R$, Lobanenkov $V$ : CTCF is a uniquely versatile transcription regulator linked to epigenetics and disease. Trends Genet 2001, 17(9):520-527.

40. Essien K, Vigneau S, Apreleva S, Singh LN, Bartolomei MS, Hannenhalli S: CTCF binding site classes exhibit distinct evolutionary, genomic, epigenomic and transcriptomic features. Genome Biol 2009, 10(11):R131.
41. Berman BP, Weisenberger DJ, Aman JF, Hinoue T, Ramjan Z, Liu Y, Noushmehr $\mathrm{H}$, Lange CP, van Dijk CM, Tollenaar RA, et al: Regions of focal DNA hypermethylation and long-range hypomethylation in colorectal cancer coincide with nuclear lamina-associated domains. Nat Genet 2012, 44(1):40-46

42. Guelen L, Pagie L, Brasset E, Meuleman W, Faza MB, Talhout W, Eussen BH, de Klein A, Wessels $L$, de Laat W, et al: Domain organization of human chromosomes revealed by mapping of nuclear lamina interactions. Nature 2008, 453(7197):948-951.

43. Hansen KD, Timp W, Bravo HC, Sabunciyan S, Langmead B, McDonald OG, Wen B, Wu H, Liu Y, Diep D, et al: Increased methylation variation in epigenetic domains across cancer types. Nat Genet 2011, 43(8):768-775.

44. Bert T, Lubomierski N, Gangsauge S, Munch K, Printz H, Prasnikar N, Robbel C, Simon B: Expression spectrum and methylation-dependent regulation of melanoma antigen-encoding gene family members in pancreatic cancer cells. Pancreatology 2002, 2(2):146-154.

45. Lim JH, Kim SP, Gabrielson E, Park YB, Park JW, Kwon TK: Activation of human cancer/testis antigen gene, XAGE-1, in tumor cells is correlated with CpG island hypomethylation. Int J Cancer: Journal International du Cancer 2005, 116(2):200-206.

46. Witcher M, Emerson BM: Epigenetic silencing of the p16(INK4a) tumor suppressor is associated with loss of CTCF binding and a chromatin boundary. Mol Cell 2009, 34(3):271-284.

47. Qiu X, Hother C, Ralfkiaer UM, Sogaard A, Lu Q, Workman CT, Liang G, Jones PA, Gronbaek K: Equitoxic doses of 5-azacytidine and 5-aza -2'deoxycytidine induce diverse immediate and overlapping heritable changes in the transcriptome. PLoS One 2010, 5(9):10.

48. Akers SN, Odunsi K, Karpf AR: Regulation of cancer germline antigen gene expression: implications for cancer immunotherapy. Future Oncol 2010, 6(5):717-732.

49. Gjerstorff MF, Burns J, Ditzel HJ: Cancer-germline antigen vaccines and epigenetic enhancers: future strategies for cancer treatment. Expert Opin Biol Ther 2010, 10(7):1061-1075.

doi:10.1186/1471-2407-13-144

Cite this article as: Kim et al:: Derepression of Cancer/Testis Antigens in cancer is associated with distinct patterns of DNA Hypomethylation. BMC Cancer 2013 13:144.

\section{Submit your next manuscript to BioMed Central and take full advantage of:}

- Convenient online submission

- Thorough peer review

- No space constraints or color figure charges

- Immediate publication on acceptance

- Inclusion in PubMed, CAS, Scopus and Google Scholar

- Research which is freely available for redistribution

Submit your manuscript at www.biomedcentral.com/submit
C) Biomed Central 